with no PC, the $79(15.8 \%)$ patients with PC involvement had a higher one year mortality rate $(70.9 \%$ vs. $18.8 \%, \mathrm{p}<0.0001)$, more ED visits/year for HF $(0.82$ vs. $0.52, \mathrm{p}<0.0001)$, and more hospital admissions/year for HF $(1.4$ vs. $0.85, \mathrm{p}<0.0001)$. Using the heart failure palliative care score criteria, 60 patients had scores $>=2$. Compared to those with scores $<2$, these patients had a higher 1-year mortality rate $(50 \%$ vs. $24 \%, \mathrm{p}<0.0001)$ and more ED visits/year for $\mathrm{HF}(0.83$ vs. $0.54, \mathrm{p}<0.01)$. Only $40.0 \%$ of these high risk patients had any PC involvement. Conclusion: We found that few HF patients had PC services involved in their care. Using this novel HF palliative care referral score, we were able to identify patients with a significantly greater risk of mortality and morbidity. This study provides evidence that the ED is an appropriate setting to identify and refer high risk HF patients who would likely benefit from earlier PC involvement and may be a future avenue for PC access for these patients.

Keywords: palliative care, heart failure, emergency department

\section{LO03 \\ Application and usefulness of outpatient cardiac testing among emergency department patients with syncope}

O. Cook, BHSc, M.A. Mukarram, MBBS, MPH, S. Kim, BScH, K. Arcot, MSc, M. Taljaard, PhD, M. Sivilotti, MSc, MD, B.H. Rowe, MD, MSc, V. Thiruganasambandamoorthy, MD, MSc, University of Ottawa, Department of Emergency Medicine, Ottawa, ON

Introduction: $2.6 \%$ of emergency department (ED) syncope patients will have underlying cardiac serious conditions (e.g. arrhythmia, serious structural heart disease) identified within 30-days of disposition. If those at risk are discharged home, outpatient cardiac testing can detect underlying arrhythmias and structural heart disease, and thereby improve patient safety. We describe the frequency of outpatient referrals for cardiac testing and the proportion of cardiac serious adverse events (SAE) among high risk and non-high (low and medium) risk ED syncope patients, as defined by the Canadian Syncope Risk Score (CSRS). Methods: We conducted a multicenter prospective cohort study to enroll adult syncope patients across five large tertiary care EDs. We collected demographics, medical history, disposition, CSRS value, outpatient referrals and testing results (holter, echocardiography), and cardiac SAE. Adjudicated 30-day SAE included death due to unknown cause, myocardial infarction, arrhythmia, and structural heart disease. We used descriptive analysis. Results: Of 4,064 enrolled patients, a total of 955 patients (23\%) received an outpatient referral (mean age 57.7 years, $52.1 \%$ female). Of the 299 patients (7\%) hospitalized, 154 received outpatient cardiac testing after discharge. Among the 3,765 patients discharged home from the ED, $40 \%$ of the non-high risk patients $(305 / 756)$ and $56 \%$ of the high risk patients $(25 / 45)$ received outpatient cardiac testing. Of all patients who received outpatient cardiac testing, 4 patients $(0.8 \%)$ had serious cardiac conditions identified and all were arrhythmias. Among those with no cardiac testing, 5 patients $(0.9 \%)$ suffered cardiac SAE (80\% arrhythmias) outside the hospital. Of the $20(44 \%)$ high risk patients who did not receive outpatient cardiac testing, $2(10 \%)$ patients suffered arrhythmias outside the hospital. While among the 451 non-high risk patients, only $0.8 \%$ suffered arrhythmia outside the hospital. Conclusion: Outpatient cardiac testing among ED syncope patients is largely underutilized, especially among high risk patients. Better guidelines for outpatient cardiac testing are needed, as current practice is highly variable and mismatched with patient risk.

Keywords: cardiac, syncope, resource utilization

\section{LO04}

Very low concentrations of high-sensitivity troponin $T$ at presentation can rapidly exclude acute myocardial infarction in a significant proportion of ED chest pain patients

J. Andruchow, MD, MSc, A. McRae, MD, T. Abedin, MSc, D. Wang, MSc, E. Lang, MD, G. Innes, MD, University of Calgary, Calgary, AB

Introduction: Chest pain is one of the most common presenting complaints to emergency departments (EDs) across the world, and the exclusion of acute myocardial infarction (AMI) using troponin testing is central to the care of many of these patients. Testing strategies using conventional troponin assays require repeat testing over many hours to avoid missed diagnoses. This study aims to validate the ability of very low concentrations of troponin at presentation to exclude AMI in ED chest pain patients. Methods: This prospective cohort study was conducted at a single urban tertiary centre and regional percutaneous coronary intervention site in Calgary, Alberta. Patients were eligible for enrolment if they presented to the ED with chest pain, were 25-years or older and required biomarker testing to rule out AMI at the discretion of the attending emergency physician. Patients were excluded if they had clear acute ischemic ECG changes, new arrhythmia or renal failure requiring hemodialysis. High-sensitivity troponin-T (Roche Elecsys hs-cTnT) results were obtained in all patients at presentation. Relevant outcomes were obtained from administrative data. The primary outcome was AMI within 30-days of ED visit, the secondary outcome was 30-day major adverse cardiac events (MACE). The study was REB approved. Results: A total of 1,016 patients were enrolled from August 2014-September 2016, of which 174 (17.1\%) patients had an initial troponin below the limit of blank $(<3 \mathrm{ng} / \mathrm{L})$ and 369 (36.3\%) had a level below the limit of detection $(<5 \mathrm{ng} / \mathrm{L})$. The sensitivity and negative predictive value (NPV) of a troponin below limit of blank $(<3 \mathrm{ng} / \mathrm{L})$ for 30-day AMI were $100 \%$ (95\% CI $89.3 \%-100 \%$ ) and $100 \%$ (95\% CI 97.8-100\%), respectively. The sensitivity and NPV of a troponin below limit of detection ( $<5 \mathrm{ng} / \mathrm{L}$ ) for 30-day AMI were 93.8\% (95\% CI 80.098.3\%) and $99.5 \%$ (95\% CI 98.1-99.9\%) respectively. Sensitivity for 30-day MACE at both cutoffs was lower: 96.1\% (95\% CI 92.5-98.0\%) for $<3 \mathrm{ng} / \mathrm{L}$, and $88.4 \%$ (95\% CI 83.3-92.1\%) for $<5 \mathrm{ng} / \mathrm{L}$, respectively. Conclusion: A high sensitivity troponin $\mathrm{T}$ result below the limit of blank is highly sensitive at excluding AMI and identifies patients at reasonably low risk of 30-day MACE. A result below the limit of detection will identify a larger population of patients as low risk but has a greater risk of missed AMI and MACE.

Keywords: chest pain, troponin, myocardial infarction

\section{LO05}

In patients presenting to the ED with STEMI, is the provision of morphine associated with worse patient outcomes?

D. Barbic, MD, MSc, F.X. Scheuermeyer, MD, Q. Salehmohamed, BSc, B. Kim, BSc, S. Barbic, PhD, T. Kawano, MD, B.E. Grunau, MD, J. Christenson, MD, University of British Columbia, Vancouver, BC

Introduction: ST-elevation myocardial infarction (STEMI) presenting to the ED is a significant health burden. The provision of IV morphine with doses titrated to provide comfort is recommended in the AHA STEMI Guidelines, yet there is limited evidence of safety in this setting. The primary objective of this study was to measure potential harm associated with the provision of IV morphine in STEMI patients presenting to the ED. Methods: This was a two centre retrospective chart review from an urban, inner city, academic ED with an annual census of 85,000 visits, and an affiliated community hospital with 35,000 annual visits. Consecutive patients from April 2009 to January 
2015 presenting to the 2 EDs with a diagnosis of STEMI were identified in the ED database. Eight trained research assistants, blinded to the study hypothesis, used standardized data collection templates. The primary investigator double collected $20 \%$ of all data to ensure completeness and accuracy. Results: We included 311 patients with STEMI (124 received morphine [M]; 187 no morphine [nM]). The ages of the two groups were similar (mean $64 \mathrm{yrs}[\mathrm{M}] \& 67 \mathrm{yrs}[\mathrm{nM}]$; median 63 yrs $[\mathrm{M}]$ \& $66 \mathrm{yrs}[\mathrm{nM}]$; IQR 45-81 [M] and 45.5-86.5 [nM]); as were the proportion of female patients $(21.0 \%[\mathrm{M}] \& 23.5 \%[\mathrm{nM}]$. The pre-STEMI Charlson comorbidity scores (mean 2.6), median time to first ECG (11 $\min [\mathrm{M}] \& 16 \mathrm{~min}[\mathrm{nM}]$ ), and mean time-to-needle for PCI (96.8 min [M] \& $92.0 \min [\mathrm{nM}])$ were similar between groups. The mean CCU length of stay (LOS) (9.3 days vs 6.3 days) and hospital LOS (7.4 days vs 4.6 days) were longer for patients receiving morphine than those not receiving morphine. Rates of congestive heart failure, acute kidney injury and cardiac arrest in hospital were unchanged between the groups. Unadjusted mortality was similar $(10.5 \%[\mathrm{M}]$ vs $13.3 \%[\mathrm{nM}]$ ) between groups. Binary logistic regression controlling for age, Charlson score, first and peak troponin values demonstrated an association between receiving morphine in the ED and an increased risk of death at 30 days (OR 8.1; 95\% CI 7.1.-9.1). Conclusion: The provision of morphine to patients with STEMI in the ED may be associated with increased CCU and hospital LOS. When controlling for age, pre-STEMI Charlson score, first and peak troponin values, receiving morphine was associated with an increased risk of death at 30 days. Further research to elucidate this association is warranted.

Keywords: acute myocardial infarction, morphine, mortality

\section{LO06}

Role of the age adjusted D-dimer in suspected deep venous thrombosis P. Reardon, MD, S. Patrick, BSc, M. Taljaard, PhD, K. Thavorn, PhD, M.A. Mukarram, MBBS, MPH, S. Kim, BScH, G. Le Gal, MD, PhD, V. Thiruganasambandamoorthy, MD, MSc, Department of Emergency Medicine, University of Ottawa, Ottawa, ON

Introduction: It is well established that a negative D-dimer will reliably rule out thromboembolism in selected low risk patients. Multiple modified D-dimer cutoffs have been suggested for older patients to improve diagnostic specificity. However, these approaches are better established for pulmonary embolism than for deep venous thrombosis (DVT). This study will evaluate the diagnostic performance of previously suggested D-dimer cutoffs for low risk DVT patients in the ED, and assess for a novel cutoff with improved performance. Methods: This health records review included patients $>50$ years with suspected DVT who were low-risk and had a D-dimer performed. Our analysis evaluated the diagnostic accuracy of D-dimer cutoffs of 500 and the age adjusted (age x 10) rule for patients $>50$ years; and 750, and 1,000 cutoffs for patients $>60$ years. 30-day outcome was a diagnosis of DVT. We also assessed the diagnostic accuracy for a novel cutoff (age x 12.5). Results: 1,000 patients (mean age 68 years; $59 \%$ female) were included. Of these, 110 patients $(11 \%)$ were diagnosed with DVT. The conventional cutoff of $<500 \mu \mathrm{g} / \mathrm{L}$ demonstrated a sensitivity of $99.1 \%$ (95\% CI 95.0-99.9) and a specificity of $36.4 \%$ (95\% CI 33.2-39.7). For patients $>60$ years, the absolute cutoffs of 750 and 1,000 showed sensitivity of $98.7 \%$ (95\% CI, 92.9, 99.9), and the specificity increased to $48.6 \%$ (95\% CI, 44.5-52.8\%) and 62.1\% (95\% CI, 58.1-66.1\%) respectively. For all study patients, age adjusted D-dimer demonstrated a sensitivity of $99.1 \%$ (95\% CI 95.0-99.9) and a specificity of 51.2\% (95\% CI, 47.954.6). A novel age adjusted cutoff (age $x$ 12.5) for patients $>50$, demonstrated a sensitivity of $97.3 \%$ (95\% CI 92.2-99.4) and a specificity of $61.2 \%$ (95\% CI 58.0-64.5). When compared to conventional cutoff, the age adjusted cutoffs (age x 10 and age x 12.5) would have resulted in an absolute decrease in further investigations of $13.1 \%$ and $22.2 \%$, respectively, with false negative rates of $0.1 \%$ and $0.3 \%$. Conclusion: Among older patients with suspected DVT and low clinical probability, the age adjusted D-dimer increases the proportion of patients among whom DVT can be ruled out. A novel cutoff (age x 12.5) demonstrated improved specificity. Future large scale prospective studies are needed to confirm this finding and to explore the cost savings of these approaches.

Keywords: deep venous thrombosis, D-dimer

\section{LO07}

Does point of care ultrasonography improve diagnostic accuracy in emergency department patients with undifferentiated hypotension? The first Sonography in Hypotension and Cardiac Arrest in the Emergency Department (SHOC-ED1) Study; an international randomized controlled trial

M. Peach, MD, J. Milne, D. Lewis, MBBS, L. Diegelmann, MD, H. Lamprecht, MBChB, M. Stander, MBBCh, MMed EM, D. Lussier, MD, C. Pham, MD, R. Henneberry, MD, J. Fraser, BN, M. Howlett, MD, J. Mekwan, MD, B. Ramrattan, MD, J. Middleton, MD, D.J. van Hoving, MD, D. Fredericks, MD, L. Taylor, MD, T. Dahn, MD, S.T. Hurley, BSc, K. MacSween, BSc, C. Cox, MD, L. Richardson, MD, O. Loubani, BSc, MD, G. Stoica, Phd, S. Hunter, BSc, P. Olszynski, MD, P.R. Atkinson, MD, Dalhousie University, Integrated Family/Emergency Residency Program, Saint John, NB

Introduction: Point of care ultrasonography (PoCUS) is an established tool in the initial management of hypotensive patients in the emergency department (ED). It has been shown rule out certain shock etiologies, and improve diagnostic certainty, however evidence on benefit in the management of hypotensive patients is limited. We report the findings from our international multicenter RCT assessing the impact of a PoCUS protocol on diagnostic accuracy, as well as other key outcomes including mortality, which are reported elsewhere. Methods: Recruitment occurred at 4 North American and 3 Southern African sites. Screening at triage identified patients $(\mathrm{SBP}<100 \mathrm{mmHg}$ or shock index $>1$ ) who were randomized to either PoCUS or control groups. Scans were performed by PoCUS-trained physicians. Demographics, clinical details and findings were collected prospectively. Initial and secondary diagnoses were recorded at 0 and 60 minutes, with ultrasound performed in the PoCUS group prior to secondary assessment. Final chart review was blinded to initial impressions and PoCUS findings. Categorical data was analyzed using Fishers two-tailed test. Our sample size was powered at $0.80(\alpha: 0.05)$ for a moderate effect size. Results: 258 patients were enrolled with follow-up fully completed. Baseline comparisons confirmed effective randomization. The perceived shock category changed more frequently in the PoCUS group 20/127 (15.7\%) vs. control 7/125 (5.6\%); RR 2.81 (95\% CI 1.23 to $6.42 ; \mathrm{p}=0.0134$ ). There was no significant difference in change of diagnostic impression between groups PoCUS 39/123 (31.7\%) vs control 34/124 (27.4\%); RR 1.16 (95\% CI 0.786 to $1.70 ; \mathrm{p}=0.4879)$. There was no significant difference in the rate of correct category of shock between PoCUS (118/ 127; 93\%) and control (113/122; 93\%); RR 1.00 (95\% CI 0.936 to 1.08 ; $\mathrm{p}=1.00$ ), or for correct diagnosis; PoCUS 90/127 (70\%) vs control 86/122 (70\%); RR 0.987 (95\% CI 0.671 to $1.45 ; \mathrm{p}=1.00$ ). Conclusion: This is the first RCT to compare PoCUS to standard care for undifferentiated hypotensive ED patients. We found that the use of PoCUS did change physicians' perceived shock category. PoCUS did not improve diagnostic accuracy for category of shock or diagnosis.

Keywords: point of care ultrasound (PoCUS), hypotension, diagnosis 